\title{
Que Soluções para o Financiamento da Inovação?
}

\author{
What Solutions for the Financing of Innovation?
}

\author{
Maria da Conceição Constantino PORTELA ${ }^{1}$, Adalberto Campos FERNANDES ${ }^{2}$ \\ Acta Med Port 2015 Jul-Aug;28(4):419-420
}

Palavras-chave: Alocação de Recursos; Prioridades em Saúde; Racionalização de Cuidados de Saúde; Tecnologia Biomédica.

Keywords: Biomedical Technology; Health Care Rationing; Health Priorities; Resource Allocation.

Os modelos de financiamento das tecnologias de saúde têm vindo a tornar-se obsoletos. Este facto é reconhecido tanto pelos financiadores públicos - que detêm o poder de monopsónio associado em exclusivo ao financiamento público, como pela indústria farmacêutica - que detém o poder de monopólio conferido pelas patentes atribuídas aos medicamentos que desenvolve.

Estão ancorados na eficácia e nos custos, não incorporando as demais dimensões do valor. Encontram-se desajustados dos modelos de autorização de introdução no mercado (AIM), bem como da incerteza associada à eficácia, efetividade, e segurança, e ainda da evolução que tem percorrido os sistemas de saúde.

Acresce que os resultados em saúde são de magnitude diferente da que era usual: ganhos marginais, ou pelo contrário, resultados disruptivos. Por acréscimo, a qualidade de vida passou a integrar as prioridades estabelecidas, e os marcadores de eficácia já não são apenas de resultados finais, mas também de resultados intermédios, surgindo ainda os marcadores de efetividade.

Propõe-se a transição do modelo atual centrado nas lacunas terapêuticas, para um outro centrado no valor, na linha da proposta da Organização Mundial de Saúde. ${ }^{1}$

Para a indústria farmacêutica, a elevada incerteza e os custos associados ao desenvolvimento regulamentar reduzem o retorno do investimento que se manteve estável durante largas décadas, incentivando o mercado farmacêutico a evoluir. Em 1975 a despesa com investigação e desenvolvimento (I\&D) representava $9 \%$ do volume de vendas, tendo aumentado para $17 \%$ em $2010 .^{2}$ Contudo, este esforço não foi acompanhado pelo incremento no número de moléculas desenvolvidas ou aprovadas pelas autoridades reguladoras. ${ }^{2}$ Nesse intervalo de tempo observou-se um aumento do número de fusões e aquisições, e a emergência de novos modelos de negócio, focalizados na captação externa de valor. ${ }^{2}$ Neste contexto, os preços dos medicamentos aprovados por procedimento centralizado tendem a ser $70 \%$ superiores aos aprovados por procedimento descentralizado, ou nacional, sendo mais elevados quanto maior a idade dos concorrentes, e independentes do grau de inovação. ${ }^{3}$

Por outro lado, as tecnologias de saúde possuíam uma intervenção padronizada, transversal a toda a população, mas agora têm vindo a focalizar-se em segmentos populacionais com genótipos específicos, que permitem antecipar o sucesso terapêutico. Também os agentes da decisão já não estão cingidos às autoridades reguladoras e financiadoras, e à indústria farmacêutica, alargando-se o leque no sentido de uma inclusão mais vasta dos representantes da sociedade civil.

Neste contexto, a inovação deve ser perspetivada concedendo a primazia a uma visão transversal, em que os resultados das tecnologias de saúde inovadoras vão moldar os processos em que são incorporadas, e são moldados pelos agentes que integram a gestão dessas tecnologias. No seio de um processo em que deve ser possível identificar como é que cada indivíduo é agente de valor para o sistema. ${ }^{4}$

Por isso há que apostar na consistência entre os instrumentos de regulação, que contribuem para potenciar resultados e minimizar desperdícios. Apostar na transparência, que é garantia de credibilidade, e de investimento rigoroso; através da divulgação junto dos agentes económicos da inovação que o Estado quer financiar para o sistema de saúde. Apostar na proximidade, i.e., atuar sobre as desigualdades, proporcionando respostas terapêuticas tendencialmente personalizadas.

E porque não antecipar o futuro? A recente evolução do sistema de farmacovigilância proporciona um acompanhamento mais próximo da evolução do rácio risco benefício dos medicamentos, e a subsequente possibilidade de tradução no ajustamento dos preços, que represente o valor atual dos medicamentos.

Com a adoção dos novos modelos de autorização de introdução no mercado (AIM), a estrutura de custos alterou-se. Observa-se uma modificação nos custos de desenvolvimento, associada a uma maior incerteza, e incremento do tempo de comercialização sob patente. Em ordem à

\footnotetext{
1. Centro de Investigação Interdisciplinar em Saúde. Instituto de Ciências da Saúde. Universidade Católica Portuguesa. Lisboa. Portugal.

2. Gestão em Saúde e de Políticas de Saúde. Escola Nacional de Saúde Pública. Universidade Nova de Lisboa. Lisboa. Portugal.

$\triangle$ Autor correspondente: Maria da Conceição Constantino Portela. mccportela@gmail.com

Recebido: 18 de Junho de 2015 - Aceite: 18 de Junho de 2015 | Copyright @ Ordem dos Médicos 2015
} 
criação de valor o sistema de financiamento pode gerir uma partilha de risco, como está plasmado no novo modelo de avaliação de tecnologias de saúde - SiNATS - vigente em Portugal.

O segmento de mercado dos medicamentos personalizados tem vindo a ampliar-se. Sendo difícil obter dados robustos de ensaios clínicos, torna-se maior a incerteza na eficácia e efetividade associada, e por isso a identificação robusta do valor.

Quanto ao quadro regulamentar europeu questiona-se a atualidade do princípio da subsidiariedade, num quadro de autorizações centralizadas, avaliações centralizadas, centros de referência europeus, e regulação da mobilidade de doentes no espaço europeu. Seria eventualmente oportuno equacionar um sistema europeu de financiamento do valor das tecnologias de saúde, em modelo de parceria entre os Estados Membros.

Também os centros europeus de referência serão centros de excelência na prestação de cuidados de saúde, e preferenciais para a fase de desenvolvimento de novos medicamentos. Com a centralização da informação e da expertise, possuirão tendencialmente uma casuística mais favorável para um desempenho de qualidade superior, promovendo um fluxo preferencial de doentes nesse sentido. Por isso é de indubitável importância que venham a ser instituídos em Portugal, gerando um fluxo de doentes equilibrado e sustentável no espaço europeu.

Depois, a avaliação centralizada das tecnologias de saúde vai contribuir para que os medicamentos venham a ser comercializados em tempos mais próximos, e por isso o modelo de referenciação internacional de preços tenderá a apresentar-se desajustado.

Antecipar o futuro é um desafio que cria valor. Através da regulação pró-ativa, recorrendo à Academia para a apresentação de informação estratégica. Na clarificação de objetivos, e identificação de marcadores de resultados em saúde, em sede de financiamento. Focalizados em resultados que sejam do interesse dos doentes, ${ }^{5}$ e minimizando custos desnecessários. ${ }^{6} \mathrm{Na}$ decisão, considerar que a melhor decisão pode divergir da decisão mais eficiente, e que o valor social nem sempre se compagina com o valor individual. $^{7}$

\section{REFERÊNCIAS}

1. World Health Organization Regional Office for Europe. Access to new medicines in Europe: technical review of policy initiatives and opportunities for collaboration and research. Copenhagen: WHO; 2015.

2. Gleadle P, Parris S, Shipman A, Simonetti R. Restructuring and innovation in pharmaceuticals and biotechs: the impact of financialisation. Crit Pers Account. 2014;25:67-77.

3. Puig-Junoy J, López-Valcárcel BG. Launch prices for new pharmaceuticals in the heavily regulated and subsidized Spanish market, 1995-2007. Health Policy. 2014;116:170-81.

4. Edwards PJ. Value networks identify innovation in 21st century pharmaceutical research. Drug Discov Today. 2009;14:68-77.

5. Godlee F. Outcomes that matter to patients. BMJ. 2012;344:e318.

6. Brody $\mathrm{H}$. From an ethics of rationing to an ethics of waste avoidance. $\mathrm{N}$ Engl J Med. 2012;366:1949-51.
Subentendem-se novos paradigmas. A sustentabilidade advém do equilíbrio, quando se conciliam objetivos entre a oferta e a procura, decorrente de uma visão agregada do sistema de saúde, em que a integração da inovação deve fomentar a homeostasia.

O valor é a medida da inovação. Por isso as decisões estratégicas que integram o financiamento devem ser informadas pela evidência dinâmica, que favorece a respetiva aceitabilidade. ${ }^{8}$ Neste domínio a utilização dos QALYs (anos de vida ajustados pela qualidade) deve considerar as preferências sociais. ${ }^{9}$

Os sistemas de financiamento devem acomodar a inovação - das tecnologias de saúde, dos agentes sociais, e dos económicos - a partir dos resultados de valor que geram para o mesmo sistema; mas não assumir de forma cega o risco das decisões que cada uma das partes toma individualmente. Devem ser atualizados por fluxos nos dois sentidos - entradas e saídas, complementados por retrações ou incrementos no financiamento.

Deve haver lugar para preços adaptativos ao longo do ciclo de vida do medicamento, ancorados na patente, mas também no eixo da segurança. Perspetivando não só o valor de uma tecnologia para uma patologia, mas também o valor integrado das mesmas para cada doente.

A avaliação sistemática do rácio custo efetividade deve incidir também sobre a regulação. ${ }^{10} \mathrm{E}$ ser considerada a oportunidade da análise de decisão multiatributos. ${ }^{11}$

Há soluções para o financiamento da inovação. Mediadas por um Estado inovador, que fomente a oferta e a procura do valor acrescentado, e por sistemas de financiamento em recriação permanente, que refletem a natureza evolutiva do valor. Aquele que gera sustentabilidade, eficiência, e qualidade para o Sistema de Saúde.

\section{CONFLITOS DE INTERESSE}

Os autores declaram não ter quaisquer conflitos de interesse relativamente ao presente artigo.

\section{FONTES DE FINANCIAMENTO}

Este trabalho não recebeu nenhuma contribuição de subsídio ou bolsa.

7. Johri M. Can cost effectiveness analysis integrate concerns for equity? Systematic review. Int J Technol Assess Health Care. 2012;28:1-8.

8. Stafinski T, Menon D, McCabe C, Philippon DJ. To fund or not to fund Development of a decision making framework for the coverage of new health technologies. Pharmacoeconomics. 2011;29:771-80.

9. Drummond M, Tarricone R, Torbica A. Assessing the added value of health technologies:reconciling different perspectives. Value Health. 2013;16:S7-13.

10. Bouvy JC, Ebbers HC, Schellekens H, Koopmanschap MA. The costeffectiveness of Periodic Safety Update Reports for Biologicals in Europe. Clin Pharmacol Ther. 2013;93:433-42.

11. Baltussen R, Youngkong S, Paolucci F, Niessen L. Multi criteria decision analysis to prioritize health interventions: capitalizing on first experiences. Health Policy. 2010;96:262-4. 


\section{Que Soluções para o Financiamento da Inovação?}

Acta Med Port 2015:28:419-420

Publicado pela Acta Médica Portuguesa, a Revista Científica da Ordem dos Médicos

Av. Almirante Gago Coutinho, 151

1749-084 Lisboa, Portugal.

Tel: +351218428215

E-mail: submissao@actamedicaportuguesa.com

www.actamedicaportuguesa.com

ISSN:0870-399X | e-ISSN: 1646-0758

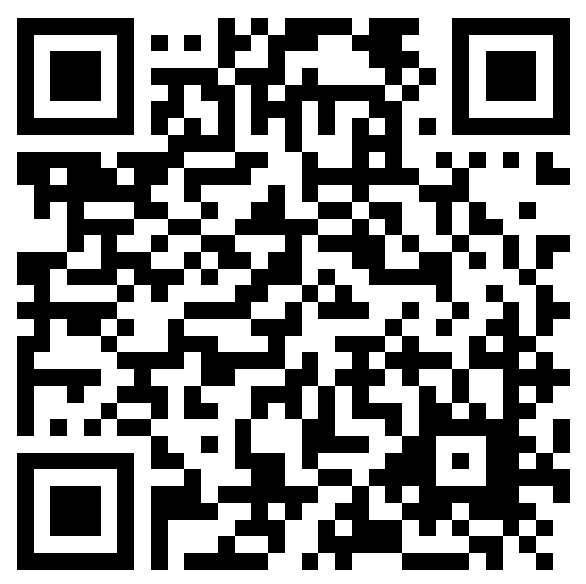

